\title{
Preparation and electronic structure study of a topological crystalline insulator, SnTe
}

Cite as: AIP Conference Proceedings 2115, 030343 (2019); https://doi.org/10.1063/1.5113182

Published Online: 12 July 2019

Arindam Pramanik, Ram Prakash Pandeya, Sangeeta Thakur, A. Thamizhavel, and Kalobaran Maiti

\section{ARTICLES YOU MAY BE INTERESTED IN}

Surface and bulk core level study of PdTe using HAXPES

AIP Conference Proceedings 2115, 030339 (2019); https://doi.org/10.1063/1.5113178

Revealing surface-state transport in ultrathin topological crystalline insulator SnTe films APL Materials 7, 051106 (2019); https://doi.org/10.1063/1.5096279

Growth and characterization of snte crystalline topological insulator

AIP Conference Proceedings 2162, 020126 (2019); https://doi.org/10.1063/1.5130336

Challenge us.

What are your needs for periodic signal detection?

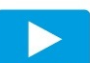

Watch

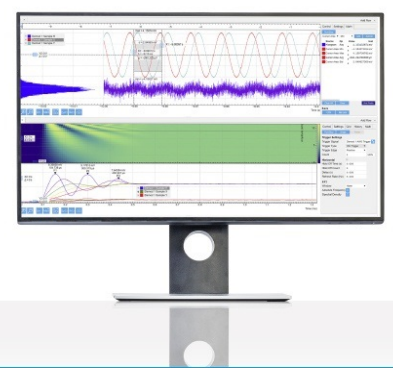

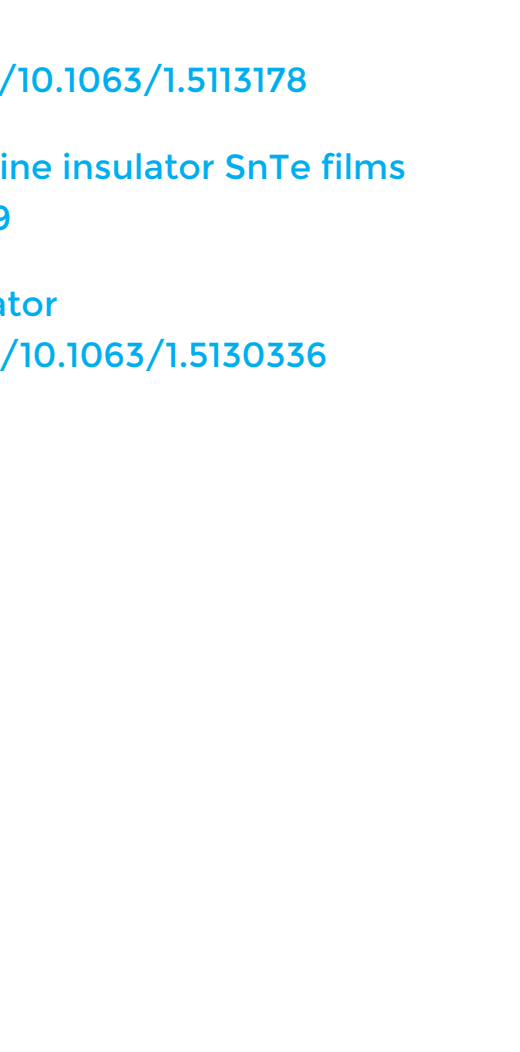




\title{
Preparation and electronic structure study of a topological crystalline insulator, SnTe
}

\author{
Arindam Pramanik, Ram Prakash Pandeya, Sangeeta Thakur, A. Thamizhavel, and \\ Kalobaran Maitia) \\ Department of Condensed Matter Physics and Materials Science, Tata Institute of Fundamental Research, Homi \\ Bhabha Road, Colaba, Mumbai, 400005, India. \\ ${ }^{a}$ Corresponding author: kbmaiti@tifr.res.in
}

\begin{abstract}
In this paper, we report preparation and characterization of high quality single crystals of a topological crystalline insulator, SnTe. Samples were prepared using modified Bridgman method and were characterized by powder diffraction, Laue diffraction and energy dispersive $x$-ray diffraction method. From the resistivity measurements, the temperature for the displacive phase transition is determined to be $40 \mathrm{~K}$. Furthermore, core level photoemission of $\mathrm{Sn} 3 s$ and Te $3 p$ using photon energy of $5945.24 \mathrm{eV}$ at different temperatures shows that structural transition does not have discernible effect on the studied core level spectra. We observe intense satellite features in the core level spectra suggesting importance of electron correlation in the electronic properties of this system.
\end{abstract}

\section{INTRODUCTION}

SnTe is a narrow gap semiconductor which forms in face centered cubic (FCC) structure. Recently, both theoretical [1] and angle resolved photoemission measurements [2] have confirmed the topological nature of the surface states found in the single crystal of $\mathrm{SnTe}$. Unlike $\mathrm{Bi}_{2} \mathrm{Se}_{3}$, where time reversal symmetry protects the surface states [3], surface states in SnTe are protected by symmetry of the crystal structure [1]. Hence the name, topological crystalline insulator.

SnTe shows displacive phase transition at lower temperature [4]. High temperature FCC structure undergoes a transformation to rhombohedral structure in the low temperature region. Depending on the carrier density, this transition temperature may vary [5]. In this work, we have prepared high quality SnTe single crystal and found out the transition temperature of the sample by resistivity measurement exhibiting anomalous increase near transition point. Finally, we employed hard $x$-ray photoemission spectroscopic (HAXPES) technique to investigate the core levels of Sn and Te. Measurements were carried out at several temperatures to study the evolution of the spectra with temperature and the effect of structural transition on the core levels.

\section{EXPERIMENTAL METHODOLOGY}

High quality single crystals of SnTe were prepared and were cut to pieces of required size using wire cut EDM, which were further used for hard $x$-ray measurements. 
(a)

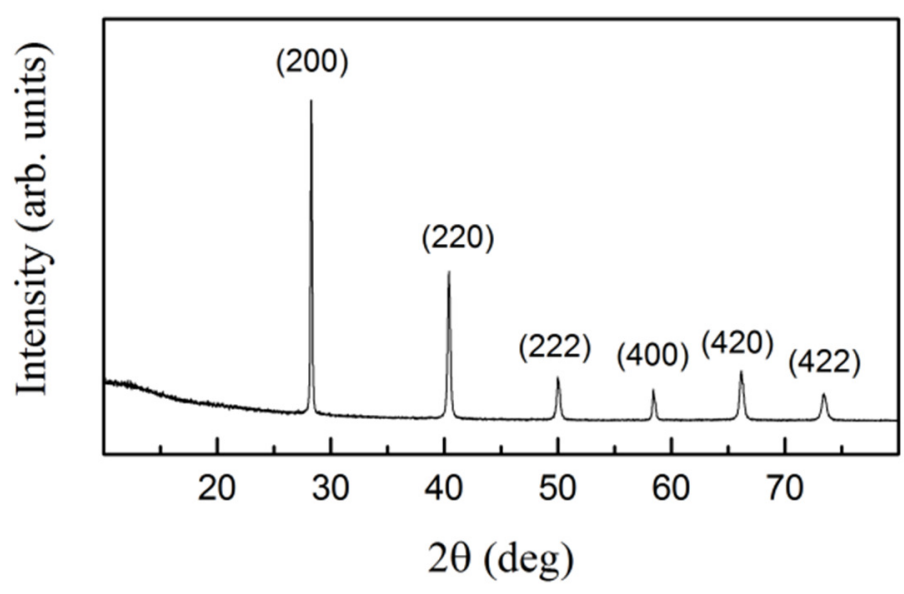

(b)

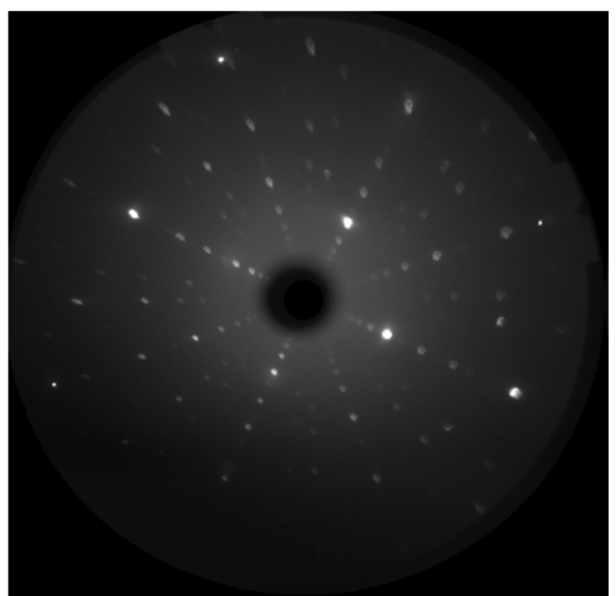

FIGURE 1. (a) Powder $x$-ray diffraction (XRD) pattern of SnTe. Miller indices of the planes giving rise to peaks are also mentioned. (a) Laue diffraction pattern of $\mathrm{SnTe}(100)$ single crystal revealing high degree of crystallinity of the sample.

Sample Preparation and Characterization: Single crystals of SnTe were prepared by modified Bridgman method. Stoichiometric mixture of $\mathrm{Sn}$ and Te was vacuum sealed in a quartz ampoule. The ampoule was heated to $900{ }^{\circ} \mathrm{C}$ in a box furnace and was kept there for 24 hours for homogenization. Then the furnace was slow cooled to $850^{\circ} \mathrm{C}$ at the rate of $5^{\circ} \mathrm{C} / \mathrm{hr}$ followed by another stage of very slow cooling to $780^{\circ} \mathrm{C}$ at the rate of $1^{\circ} \mathrm{C} / \mathrm{hr}$ (melting point of SnTe $\sim 808^{\circ} \mathrm{C}$ ). Subsequently it was taken to room temperature with a cooling rate of $60^{\circ} \mathrm{C} / \mathrm{hr}$. Prepared samples were characterized by powder $x$-ray diffraction (XRD), Laue diffraction and energy dispersive $x$-ray diffraction (EDX). Absence of any impurity peak in powder diffraction pattern shown in Fig. 1(a) ensures the singlephase nature of the sample. Sharp spots in Laue pattern shown in Figure 1(b), confirm the single crystallinity of the sample. Results of resistivity measurements are discussed in the next section.

P09 beamline, PERTA III, Hamburg, Germany. Spectrometer from Specs GmbH equipped with an electron analyzer from Specs was used for the measurements. Photon energy of $5945.24 \mathrm{eV}$ was chosen for all the experiments with an instrumental resolution of $200 \mathrm{meV}$. Low temperature was achieved using an open cycle Helium cryostat from Janis Cryogenic Research Systems, USA. Samples were cleaved in situ to expose the clean surface for the measurements. Experiments were carried out in a base vacuum better than $1 \times 10^{-10} \mathrm{mbar}$.

\section{RESULTS AND DISCUSSIONS}

Structural transition temperature was determined from resistivity measurements. Figure 2 shows the resistivity plot as a function of temperature. The data show typical metallic resistivity exhibiting gradual increase with the increase in temperature. The value of the resistivity quite small signifying good metallicity of the material. We observe a kink near 40 $\mathrm{K}$ (see arrow) indicating the structural transition; for better clarity, we plot the derivative of resistivity in the same plot and a sharp transition is evident at $40 \mathrm{~K}$ $[4,5]$. This anomalous increase of resistivity near the transition is believed to

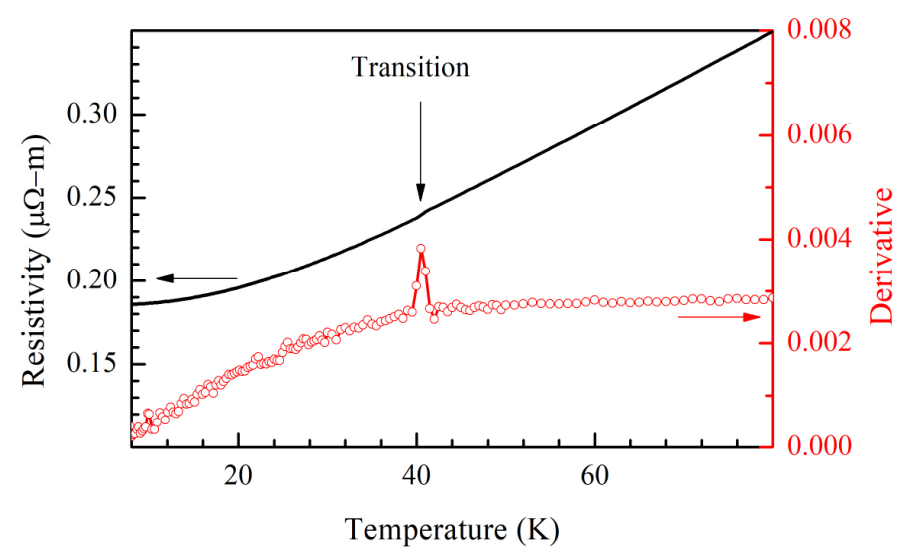

FIGURE 2. Resistivity plot shows a sudden increase near $40 \mathrm{~K}$, which 
be arising due to the interaction between charge carrier and TO-phonons [4-6].

We now study the core level spectra to investigate the change in electronic structure across the structural transition. Hard $x$-ray are used for the measurements as the escape depth of photoelectrons at this energy is high and the technique is reasonably bulk sensitive [7-9]. The experimental results are shown in Fig. 3; upper panel of the figure shows the core level spectrum of $\mathrm{Sn} 3 s$ and Te $3 p$ collected at $120 \mathrm{~K}$ in the normal emission geometry. Raw data are represented by black open circles. The peaks at $819.2 \mathrm{eV}$ and $870.1 \mathrm{eV}$ are the photoemission signals from spin-orbit split Te $3 p_{3 / 2}$ and Te $3 p_{1 / 2}$. The intense feature at $885.3 \mathrm{eV}$ appears due to $\mathrm{Sn} 3 \mathrm{~s}$ photoemission signal. In addition, there are several peaks in the spectral region shown due to the final state effect associated to photoexcitations.

In order to derive the constituent features, we have simulated the experimental spectrum with a set of asymmetric peaks. The resultant fit is shown by line superimposed on the experimental data points and exhibit excellent representation of the experimental spectrum. Asymmetry in the lineshape of the peaks have been accounted for by the use of Doniach-Sunjic line shape appearing due to low energy excitations across the Fermi level in

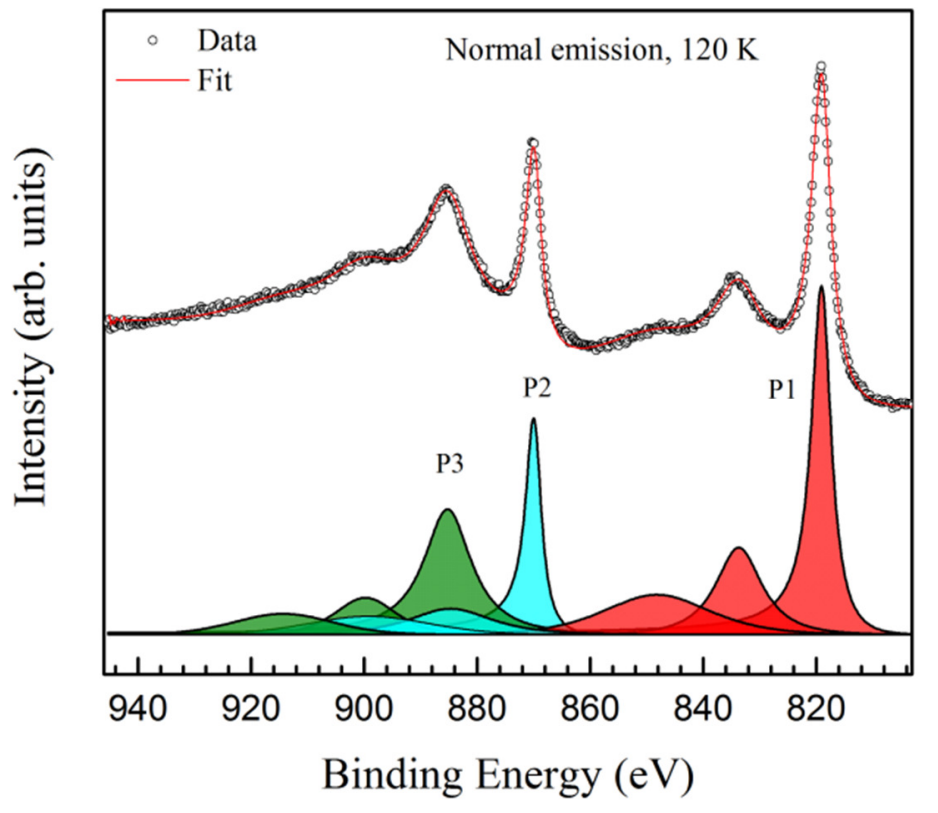

FIGURE 3. Combined spectrum of $\mathrm{Sn} 3 s$ and Te $3 p$ taken at $120 \mathrm{~K}$ in normal emission geometry. Upper panel shows the data (open circle) overlapped with the simulated spectrum. Lower panel shows the component peaks. Altogether nine peaks are seen. Te $3 p_{3 / 2}(\mathrm{P} 1)$, Te $3 p_{1 / 2}(\mathrm{P} 2)$ and $\mathrm{Sn} 3 s$ (P3) core levels have binding energies of $819.2 \mathrm{eV}, 870.1 \mathrm{eV}$ and $885.3 \mathrm{eV}$, respectively. $\mathrm{P} 1$ is accompanied by two satellite peaks, filled with red color, at higher binding energies. Similarly, P2 and P3 have satellite peaks marked with cyan and green color respectively. a metallic system. Large asymmetry of the peaks indicates presence of broad valence/conduction bands at the Fermi level. The fitting of the spectrum necessitated nine features of varying intensities, as is clear from the lower panel in Fig. 3. Peaks denoted by P1, P2 and P3 represent the photoemission of Te $3 p_{3 / 2}$, Te $3 p_{1 / 2}$ and Sn $3 s$ core levels, respectively. Each of these three peaks is accompanied by two satellite peaks positioned at higher binding energies. Satellites are situated $14.5 \mathrm{eV}$ and $29 \mathrm{eV}$ away from the main peak. Presence of such satellite peaks arising due to the final states in the photoexcitation process indicate that consideration of interaction among the charge carriers is important to capture the electronic structure of this system.

We now investigate the temperature evolution of the electronic structure across the structural transition. For that purpose, we have collected spectra at $120 \mathrm{~K}$ and $25 \mathrm{~K}$, the experimental data are shown in Fig. 4. Both the spectra are collected at normal emission geometry to make sure highest bulk sensitivity. We notice that both the spectra superimposes over one another almost exactly. This is surprising as one expects changes in Madelung potential with the structural change that will shift the peak positions. Larger phonon broadening of the peaks expected at higher temperature which is also absent - of course, the phonon effect may not be reflected in these features due to their large intrinsic width $(\sim \mathrm{eV})$ compared to the phonon energies $(\sim 100 \mathrm{meV})$. These results establish that structural change has very little effect on the local potential of $\mathrm{Sn}$ and Te in this material. If there is any change in the local potential, that is screened by the mobile charge carriers. 


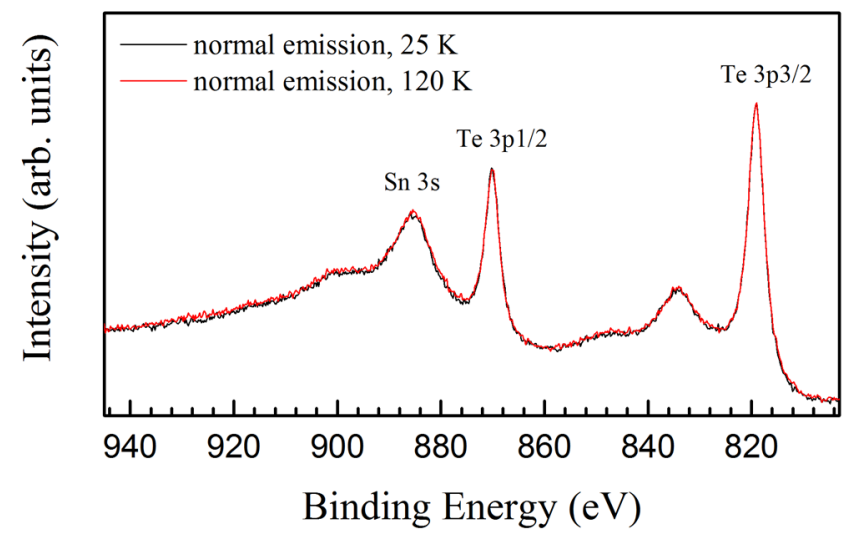

FIGURE 4. Sn $3 s$ and Te $3 p$ spectrum taken at $25 \mathrm{~K}$ and $120 \mathrm{~K}$ in normal emission geometry. Plot in red and black color show the data collected at $120 \mathrm{~K}$ and $25 \mathrm{~K}$, respectively.

\section{CONCLUSIONS}

In summary, we have successfully prepared high quality single crystals of a topological crystalline insulator, SnTe. Resistivity measurement was done to find out the structural transition temperature. Furthermore, hard $x$-ray photoemission measurements were carried out to study the core levels spectra of the bulk of the material. Spectrum collected below the transition temperature shows no discernible change from the spectrum taken above the transition temperature, which is surprising. Temperature induced broadening is also absent. We observe intense satellite features associated to the core level excitations indicating finite role of electron correlation in the electronic properties of this system.

\section{ACKNOWLEDGMENTS}

The authors acknowledge financial support from the DST-DESY project to perform the experiments at P09 beamline at PETRA III, Hamburg, Germany and Dr. Andrei Hsolkovsky for his help during the measurements.

\section{REFERENCES}

1. Timothy H. Hsieh et.al., Nat. Commun. 3, 982 (2012).

2. Y. Tanaka et. al., Nat. Phys. 8, 800-803 (2012); Su-Yang Xu et. al., Nat. Commun. 3, 1192 (2012).

3. H. Zhang et. al., Nat. Phys. 5, 438-442 (2009).

4. K. L. I. Kobayashi et. al., Solid State Commun. 17, 875-878 (1975).

5. K. L. I. Kobayashi et. al., Phys. Rev. Lett. 37, 772 (1976).

6. S. Katayama and D. L. Mills, Phys. Rev. B 22, 336 (1980).

7. K. Maiti et al., Phys. Rev. B 73, 052508 (2006).

8. S. Thakur, and K. Maiti, Solid State Commun. 193, 45 (2014).

9. K. Maiti, J. Fink, S. de Jong, M. Gorgoi, C. Lin, M. Raichle, V. Hinkov, M. Lambacher, A. Erb, and M. S. Golden, Phys. Rev. B 80, 165132 (2009). 